\title{
HERMITIAN QUADRATIC FORMS IN A QUASI-FIELD
}

\section{GORDON PALL}

1. Introduction. E. Witt ${ }^{1}$ proved the following theorem concerning quadratic forms in a fairly general field:

THEOREM 1. Let $f_{1}=a x_{1}{ }^{2}+\phi_{1}\left(x_{2}, \cdots, x_{n}\right)$ and $f_{2}=a x_{1}{ }^{2}+\phi_{2}\left(x_{2}, \cdots\right.$, $x_{n}$ ) be quadratic forms whose coefficients lie in a given field $F$ in which $2 \neq 0$. Then the equivalence in $F$ of $f_{1}$ and $f_{2}$ implies that of $\phi_{1}$ and $\phi_{2}$.

It is our purpose here to generalize this theorem to any quasi-field (a field, except that multiplication may not be commutative) on which is defined a conjugate operation of period 2 with the usual properties

$$
\overline{a+b}=\bar{a}+\bar{b}, \quad \overline{a b}=\bar{b} \cdot \bar{a} .
$$

Well known examples are any field with $\vec{a}=a$; the field of complex numbers with the usual complex conjugate; the system of quaternions with real coefficients and the usual conjugate. The analogue in a quasi-field of quadratic form in a field is the hermitian quadratic form

$$
f=\bar{x}^{\prime} A x=\sum_{i, j=2}^{n} \bar{x}_{i} a_{i j} x_{j} \text {, where } \bar{A}^{\prime}=A \text {, or } \quad \bar{a}_{i j}=a_{j i} \text {. }
$$

The scalars of a quasi-field are the elements $s$ such that $\bar{s}=s$. The diagonal elements of a hermitian matrix are therefore scalars. The process of completing squares is carried out in much the same way as in a field. Thus if, in $f$ above, $a_{11} \neq 0$,

$$
\begin{aligned}
f= & \left(\bar{x}_{1}+\sum_{i=1}^{n} \bar{x}_{i} a_{i 1} a_{11}{ }^{-1}\right) a_{11}\left(x_{1}+\sum_{i=2}^{n} a_{11}^{-1} a_{1 i} x_{i}\right) \\
& +\sum_{j, k=2}^{n} \bar{x}_{j}\left(a_{j k}-a_{j 1} a_{11}{ }^{-1} a_{1 k}\right) x_{k} .
\end{aligned}
$$

Hence the analogue of a form like $f_{1}$ in Witt's theorem can be written

$$
\bar{x}_{1} a x_{1}+\phi, \quad \text { where } \phi=\sum_{i, j=2}^{n} \bar{x}_{i} b_{i j} x_{j}, \quad b_{i j}=b_{j i} .
$$

Since determinants do not exist in a quasi-field (except for hermitian matrices), we cannot demonstrate that a matrix $T$ is nonsingular

Presented to the Society, September 17, 1945; received by the editors October 28, 1944.

${ }^{1}$ See bibliography. 
by the nonvanishing of a determinant. Instead, we may construct explicitly the reciprocal matrix $V$, such that $V T=T V=I$.

We shall, in $\S 3$, consider automorphs of $f$, that is matrices $T$ such that $\bar{T}^{\prime} A T=A$. If $z$ denotes the first column of $T$, then $\bar{z}^{\prime} A z=a_{11}$, that is $z$ is a representation of the leading coefficient $a_{11}$ of $f$. If $a_{11} \neq 0$, we shall for any given representation $z$ of $a_{11}$ construct a corresponding automorph of $f$.

2. A generalization of Witt's theorem. The theorem we shall prove is the following.

THEOREM 2. Let $F$ be a quasi-field with a conjugate operation as described above, $2 \neq 0$. Let a be a nonzero scalar, and $B_{1}, B_{2}$ nonsingular hermitian matrices of order $n-1$, with elements in $F$. Let

$$
A_{1}=\left[\begin{array}{cc}
a & 0^{\prime} \\
0 & B_{1}
\end{array}\right], \quad A_{2}=\left[\begin{array}{cc}
a & 0^{\prime} \\
0 & B_{2}
\end{array}\right] \text {. }
$$

where 0 denotes a column, and $0^{\prime}$ a row, of $n-1$ zeros. Let $T$ denote any transformation (with coefficients in $F$ ) of $A_{1}$ into $A_{2}$, that is let

$$
A_{2}=\bar{T}^{\prime} A_{1} T \text {. }
$$

Then we can construct a transformation of $B_{1}$ into $B_{2}$.

Proof. We can write (2) in the form

$$
\left[\begin{array}{ll}
a & 0^{\prime} \\
0 & B_{2}
\end{array}\right]=\left[\begin{array}{ll}
\bar{x}_{0} & \bar{x}^{\prime} \\
\bar{y} & \bar{T}_{1}^{\prime}
\end{array}\right]\left[\begin{array}{ll}
a & 0^{\prime} \\
0 & B_{1}
\end{array}\right]\left[\begin{array}{ll}
x_{0} & y^{\prime} \\
x & T_{1}
\end{array}\right],
$$

where $x_{0}$ is a constant, $x$ and $y$ are column vectors of $n-1$ components, $T_{1}$ a matrix of order $n-1$. Expanding (3) we get

$$
\begin{gathered}
\bar{x}_{0} a x_{0}+\bar{x}^{\prime} B_{1} x=a, \\
\bar{x}_{0} a y^{\prime}+\bar{x}^{\prime} B_{1} T_{1}=0^{\prime}, \quad \bar{y} a x_{0}+\bar{T}_{1}^{\prime} B_{1} x=0, \\
\bar{y} a y^{\prime}+\bar{T}_{1}{ }^{\prime} B_{1} T_{1}=B_{2} .
\end{gathered}
$$

Our problem is to derive from (4)-(6) a transformation of $B_{1}$ into $B_{2}$.

Suppose we could secure $x_{0}=1, x=0$, to begin with. Then by (5), $y^{\prime}=0^{\prime}, \bar{y}=0$; and by (6), $\bar{T}_{1}^{\prime} B_{1} T_{1}=B_{2}$. What we shall do is construct a nonsingular automorph $U$ of $A_{1}$ whose first column is the same as that of $T$. Having done this, let

$$
W=\left[\begin{array}{ll}
z_{0} & w^{\prime} \\
z & U_{1}
\end{array}\right] \text { be the reciprocal of } U=\left[\begin{array}{ll}
x_{0} & \cdot \\
x & .
\end{array}\right] .
$$

Then $z_{0} x_{0}+w^{\prime} x=1, z x_{0}+U_{1} x=0$, and so 


$$
W T=\left[\begin{array}{cc}
z_{0} & w^{\prime} \\
z & U_{1}
\end{array}\right]\left[\begin{array}{ll}
x_{0} & y^{\prime} \\
x & T_{1}
\end{array}\right]=\left[\begin{array}{ll}
1 & u^{\prime} \\
0 & V
\end{array}\right],
$$

say; and $W T$ also replaces $A_{1}$ by $A_{2}$. By the preceding remark, $\bar{V}^{\prime} B_{1} V=B_{2}$.

Hence Theorem 2 is a consequence of the following theorem; or see $\$ 4$.

3. Automorphs with an assigned first column. We now prove:

THEOREM 3. Let $A=\left(a_{i j}\right)$ be any nonsingular hermitian matrix with coefficients in a quasi-field $F$ of characteristic not 2 . Let $z$ be a representation in $F$ of $a_{11}$, that is $\bar{z}^{\prime} A z=a_{11}$, and assume $a_{11} \neq 0$. Then there exists in $F$ a nonsingular automorph of $A$ with $z$ as its first column.

We first complete squares, which amounts to applying a transformation

$$
\begin{gathered}
\bar{P}^{\prime} A P=\left[\begin{array}{ll}
a & 0^{\prime} \\
0 & B_{1}
\end{array}\right]=A_{1} \text { say, where } P=\left[\begin{array}{ll}
1 & v^{\prime} \\
0 & I
\end{array}\right], \\
P^{-1}=\left[\begin{array}{rr}
1 & -v^{\prime} \\
0 & I
\end{array}\right] .
\end{gathered}
$$

Here $a=a_{11}$, and $B_{1}$ is hermitian with $A$. We have $\bar{z}^{\prime} A z=\bar{u}^{\prime} A_{1} u$, where $z=P u$; and if we can construct an automorph $U$ of $A_{1}$ with $u$ as first column, then $P U P^{-1}$ will be an automorph of $A$ with $z$ as first column. For, the first column of $P U$ is $P u$, and multiplication on the right by $P^{-1}$ does not change the first column of $P U$.

We can therefore use the notations (4)-(6) with $B_{2}=B_{1}$. Here $x_{0}$ and $x$ are given as satisf ying (4), and it is required to find $y$ and $T_{1}$ to satisfy (5) and (6).

The cases $x_{0}=0$ and $x_{0} \neq 0$ must be distinguished.

Let $x_{0}=0$. Then $\bar{x}^{\prime} B_{1} x=a$, and we must choose $y$ and $T_{1}$ to satisfy

$$
\bar{x}^{\prime} B_{1} T_{1}=0^{\prime}, \quad \bar{y} a y^{\prime}+\bar{T}_{1}^{\prime} B_{1} T_{1}=B_{1} .
$$

The last equation can be replaced by $\left(\overline{x y^{\prime}}\right)^{\prime} B_{1}\left(x y^{\prime}\right)+\bar{T}_{1}^{\prime} B_{1} T_{1}=B_{1}$, hence by

$$
\left(\overline{x y^{\prime}+T_{1}}\right)^{\prime} B_{1}\left(x y^{\prime}+T_{1}\right)=B_{1},
$$

in view of $\left(8_{1}\right)$. Then all of (8) will hold if we put

$$
T_{1}=I-x y^{\prime}, \quad \bar{x}^{\prime} B_{1}-a y^{\prime}=0 ;
$$

the last is satisfied if $y^{\prime}=a^{-1} \bar{x}^{\prime} B_{1}$. It will be found that $y^{\prime} x=1$, and

$$
\left[\begin{array}{ll}
0 & y^{\prime} \\
x & T_{1}
\end{array}\right]\left[\begin{array}{ll}
0 & y^{\prime} \\
x & T_{1}
\end{array}\right]=\left[\begin{array}{ll}
1 & 0 \\
0 & I
\end{array}\right] \text {. }
$$


Let $x_{0} \neq 0$. Then (5) will hold if

$$
\bar{y}=-\bar{T}_{1}^{\prime} B_{1} x x_{0}^{-1} a^{-1}, \quad a y^{\prime}=-\bar{x}_{0}^{-1} \bar{x}^{\prime} B_{1} T_{1},
$$

and then (6) becomes

$$
\bar{T}_{1}^{\prime}\left(B_{1}+B_{1} x\left(\bar{x}_{0} a x_{0}\right)^{-1} \bar{x}^{\prime} B_{1}\right) T_{1}=B_{1} .
$$

We therefore try to choose a number $k$ in $F$ to satisfy

$$
\left(I+B_{1} x k \bar{x}^{\prime}\right) B_{1}\left(I+x \bar{k} \bar{x}^{\prime} B_{1}\right)=B_{1}+B_{1} x\left(\bar{x}_{0} a x_{0}\right)^{-1} \bar{x}^{\prime} B_{1} .
$$

In virtue of (4) this will be satisfied if

$$
k+\bar{k}+k\left(a-\bar{x}_{0} a x_{0}\right) \bar{k}=\left(\bar{x}_{0} a x_{0}\right)^{-1} .
$$

Here we try the substitution $k=t^{-1}$, and multiply left and right by $t$ and $\bar{t}$ to obtain

$$
\bar{t}+t+a-\bar{x}_{0} a x_{0}=t\left(\bar{x}_{0} a x_{0}\right)^{-1} \bar{t} .
$$

To satisfy this we put $t=h+\bar{x}_{0} a x_{0}$, and find

(17) $h\left(\bar{x}_{0} a x_{0}\right)^{-1} \bar{h}=a$, which holds if $h= \pm a x_{0}, t=\left(\bar{x}_{0} \pm 1\right) a x_{0}$.

Since $2 \neq 0$ in $F$ we can choose the sign to make $t \neq 0$, so that $k$ exists. We can now solve for $T_{1}$ the equations

$$
\left(I+x \bar{k} \bar{x}^{\prime} B_{1}\right) T_{1}=I=T_{1}\left(I+x \bar{k} \bar{x}^{\prime} B_{1}\right) .
$$

For if we put $T_{1}=I+x m \bar{x}^{\prime} B_{1}$, where $m$ is a constant to be determined, then (18) will hold if

$$
\bar{k}+m+\bar{k}\left(a-\bar{x}_{0} a x_{0}\right) m=0=m+\bar{k}+m\left(a-\bar{x}_{0} a x_{0}\right) \bar{k} .
$$

Noting that $\bar{k} \bar{t}=\bar{t} \bar{k}=1$ (since $k=t^{-1}$ ), we replace (19) by

$$
m^{-1}+\bar{t}+a-\bar{x}_{0} a x_{0}=0=\bar{t}+m^{-1}+a-\bar{x}_{0} a x_{0},
$$

which holds if $m^{-1}=-\bar{t}-a+\bar{x}_{0} a x_{0}=-\left(1 \pm \bar{x}_{0}\right) a$. Thus $m$ exists, and

$$
T_{1}=I+x m \bar{x}^{\prime} B_{1} \text {. }
$$

Finally we verify that the automorph so constructed is nonsingular. By (3),

$$
\left[\begin{array}{cc}
a^{-1} \bar{x}_{0} a & a^{-1} \bar{x}^{\prime} B_{1} \\
B_{1}^{-1} \bar{y} a & B_{1}^{-1} \bar{T}_{1}^{\prime} B_{1}
\end{array}\right]\left[\begin{array}{cc}
x_{0} & y^{\prime} \\
x & T_{1}
\end{array}\right]=\left[\begin{array}{ll}
1 & 0 \\
0 & I
\end{array}\right]
$$

Hence we have only to prove that ${ }^{2}$

2 The referee remarked that (23) need not be proved since it is known that if $(\alpha)(\beta)=1$, where $(\alpha),(\beta)$ are matrices with elements in a division ring, then also $(\beta)(\alpha)=1$. 


$$
\left[\begin{array}{ll}
x_{0} & y^{\prime} \\
x & T_{1}
\end{array}\right]\left[\begin{array}{ll}
a^{-1} \bar{x}_{0} a & a^{-1} \bar{x}^{\prime} B_{1} \\
B_{1}^{-1} \bar{y} a & B_{1}^{-1} \bar{T}_{1}^{\prime} B_{1}
\end{array}\right]=\left[\begin{array}{ll}
1 & 0 \\
0 & I
\end{array}\right] .
$$

Put $r=\bar{x}_{0} a x_{0}$. Using $\bar{x}^{\prime} B_{1} x=a-r$, and verifying that $m+\bar{m}+m(a-r) \bar{m}$ $=-a^{-1}$, the proof of (23) is easy. For example, $x_{0} a^{-1} \bar{x}_{0} a+y^{\prime} B_{1}^{-1} \bar{y} a=1$ if and only if $r a^{-1} r+\bar{x}^{\prime} B_{1}\left(I+x m \bar{x}^{\prime} B_{1}\right) B_{1}^{-1}\left(I+B_{1} x \bar{m} \bar{x}^{\prime}\right) B_{1} x=r$, or $r a^{-1} r+a-r+(a-r)\left(-a^{-1}\right)(a-r)=r$, or $r=r$.

One additional remark is worth making for the case where $F$ is a (commutative) field. If $K$ is any square matrix of rank 1 , it can of course be expressed as $x y^{\prime}$, where $x$ and $y$ are column vectors. The determinant of $I+K$ is readily found, since as is easily seen,

$$
\left|I+x y^{\prime}\right|=1+x^{\prime} y=1+y^{\prime} x=1+\sum x_{i} y_{i} \text {. }
$$

The reciprocal of a matrix of the type $I+h x y^{\prime}$ (where $h$ is a constant) can be found by noting that

$$
\left(I+h x y^{\prime}\right)\left(I+k x y^{\prime}\right)=I+\left\{h+k+h k y^{\prime} x\right\} x y^{\prime},
$$

and choosing $k$ to make $h+k+h k y^{\prime} x=0$.

4. An alternative construction for Theorem 2. For some purposes, it is more advantageous not to construct an automorph of $A_{1}$, but to continue the argument from (4)-(6) as follows. If $x_{0}=0, x y^{\prime}+T_{1}$ replaces $B_{1}$ by $B_{2}$. Let $x_{0} \neq 0$. Then (5) is equivalent to (12), and (6) reduces to (13) with $B_{2}$ instead of $B_{1}$ on the right. We have (14)-(17) as before, and so $T_{1}+x \bar{k} \bar{x}^{\prime} B_{1} T_{1}$ is a transformation replacing $B_{1}$ by $B_{2}$. This transformation may in certain cases be integral (in a sense which we need not discuss here) even though no integral automorph of $A_{1}$ exists with $x_{0}$ and $x$ as first column.

It should be mentioned that the preceding methods can be extended to the case where the element $a$ is replaced by a nonsingular hermitian matrix of order greater than 1 .

\section{BiBLIOGRAPHY}

1. N. Jacobson, A note on Hermitian forms, Bull. Amer. Math. Soc. vol. 46 (1940) pp. 264-268.

2. - Simple Lie algebras over a field of characteristic zero, Duke Math. J. vol. 3 (1938) pp. 534-551.

3. B. W. Jones, An extension of a theorem of Witt, Bull. Amer. Math. Soc. vol. 48 (1942) pp. 133-142.

4. C. L. Siegel, Equivalence of quadratic forms, Amer. J. Math. vol. 63 (1941) pp. $658-680$.

5. E. Witt, Theorie der quadratischen Formen in beliebigen Körper, Journal für Mathematik vol. 176 (1937) pp. 31-44.

McGill University 